\title{
STREETLIGHT OBJECTS RECOGNITION BY REGION AND HISTOGRAM FEATURES IN AN AUTONOMOUS VEHICLE SYSTEM
}

\author{
Martins E. Irhebhude, Michael Shabi and Adeola Kolawole \\ Department of Computer Science, Nigerian Defence Academy, Nigeria
}

\begin{abstract}
In this paper Streetlight object identification is addressed using the notion of image processing. An approach based on Image Processing Techniques is proposed for selection and processing of features from the images. Histogram and Region was applied on the extracted images. Histogram and Region features were then extracted and employed to train the Support Vector Machine (SVM) classifier for streetlight recognition. Experimental results shows $99.1 \%$, $84 \%$ and $100 \%$ for histogram, region features and combination of both respectively. Experimental results have proved that the proposed method is robust, accurate, and powerful in object recognition.
\end{abstract}

\section{Keywords:}

Streetlight Recognition, Autonomous Vehicles, Image Histogram Features, Region Features, Support Vector Machine

\section{INTRODUCTION}

New technologies in communication and robotics have had a substantial influence on our daily lifestyle of which transportation is no exception. In [1] many technologies have given rise to the prospect of autonomous vehicle (AV) technology which aims to reduce crashes, energy consumption, pollution, and congestion while at the same time increasing transport accessibility. The idea of driverless vehicles has been around for decades; the exorbitant costs have hindered large-scale production. Nevertheless, there has been an acceleration in the research and development efforts in the last decade to bring the idea of the AV to fruition. For example, the advent of the Google car brought AV to the spotlight [2]. Ever envisioned the significance of lighting polls on the edge of the streets?

One of many, it was reported in [3] that a car collided with a streetlight on the road. Authors in [4] points out that in this day and age road and transport has turned into a vital part of each individual. Everyone is a road user in more ways than one. The present transport framework has limited the distances however it has then again expanded the risk of life. Consistently street crashes result in loss of numerous lives and serious wounds as well. Regardless of whether it is tied in with lighting up our environment or (for security reasons, it is constantly critical to have great quality road lights. No compelling reason to say that it will end up most extreme difficult to discover routes in the dark without legitimate road lights. As a whole we know how hazardous it can be to drive at evenings if there are no legitimate streetlights [5].

Also reported in [6], two cars collided with a streetlight which led to one of the drivers being hospitalised. These useful objects (streetlights) can also be sources of concern when dealing with AV. According to [7] the progression of computational power and algorithms, the way that computers see/perceive their environment and interact with people is developing. Input devices are no longer restricted to mice, and keyboards, furthermore, we are now being able to provide input utilizing pictures, sound, fingerprint scans and other biometric information.

In [8] object detection was defined as the process of finding instances, of real-world objects such as faces, pedestrians, traffic lights, bicycles, streetlight and buildings in images or videos. Object detection algorithms normally use extracted peculiar features and learning algorithms to recognize instances of an object. It is commonly used in applications such as image retrieval, security, surveillance, and advanced driver assistance systems (ADAS) [9] [10]. Objects can be detected using a variety of models [10], including:

- Deep learning object detection

- Feature-based object detection

In [11] the process of object detection; one major concern is that object of target is obscured by other object(s). This is because the object is not clearly expressing in the image, other than that, objects which are covering each other also make the procedure more challenging where hidden item will be identified and counted and the total number will be inaccurate. In colour processing, lighting intensity may likewise affect the original colour of the object [8]. To take out all these problems, a proper image segmentation process and strategy must take into account. In object recognition, there are steps taken to achieve effective result; each picture with intended object to be detected needs to be labelled, first; the object has to be detected in the picture/frame, then they are processed as input for training the machine, to enable it correctly recognize object in any instance it occurs [12].

For clearness of presentation this article is separated into various segments; immediately following this section is section 2, which takes a look at works done in object recognition, section 3 explains the proposed methods for streetlight recognition, and flow chart of the system, section 4 discusses the various experiments to be carried out and analysis, section 5 shows the results of the various experiments performed and performance evaluation and section 6 gives conclusion and future work.

\section{LITERATURE SURVEY}

In [13], the authors used background subtraction, morphological operation and threshold methods to track objects by monitoring the changes in objects in a video frame also.

An algorithm for shading object detection and tracking in view of the thresholding level was proposed in [14]. This algorithm can be executed to track a question of any colour or shading. The same has been exhibited on a red hued telephone. At first, this red shaded telephone is recognized from a continuous picture utilizing colour threshold method. The accuracy is enhanced by morphological process, where the object to be recognized is 
signified as white leaving the background black. At that point its location is followed by getting its coordinates from the picture. This procedure can be utilized for real-time video processing.

Identifying and locating object in computerized image has turned out to be a standout amongst the most imperative applications for modern use to ease user and spare time [11]. These techniques have been developed years back however improvement of it, is still required so as to accomplish the target more effectively and precisely. Authors in [15] put forward a setup to recognize the speed breakers that lie along the streets and to hint the driver to go ahead with speed that makes him and the vehicle agreeable, the setup was composed with:

Speed breaker dimension, camera installation, lens focusing, speed breaker detection, MATLAB simulation program. A system that detects object (in this case, mango) using few methods such as colour processing and shape detection was proposed by [16]. In [17] authors proposed a system that detects speed bumps using color segmentation and knowledge base object detection. Methods to recognise and count coin from an image was proposed in [18]. Authors in [19] proposed a system for vehicle type recognition at night time using thermal image as input. The method applies the Support vector machine (SVM) classifier on CENsus Transformed histogRam Oriented Gradient (CENTROG) keeping in mind the end goal to arrange and identify people as well as vehicles. In [20] authors used image segmentation and shape based feature extraction to detect images in an email. In a similar approach [21] proposed an algorithm that recognizes license plates from cars at a gate, for example at the entrance of a parking area. The system, in light of normal PC with camcorder, gets video frames which incorporate a visible car license plate and processes them. When license plate is identified, its digits are recognized, shown on the user interface or checked against a database, region properties was utilized for character recognition.

As we can observe from literature, it is clear that different feature approaches have been used for recognising different objects at both day and night times respectively, however not known work has attempted to detect nor recognise streetlight. Therefore, in this research work, we examined features that are region and appearance based, so as to design a technique that can recognise streetlight efficiently during the day time.

\subsection{IMAGE SEGMENTATION}

Image Segmentation is a profoundly critical tool in Image examination. To recognize the object in the picture, the region of interest (ROI) detection has been applied in numerous areas, for example [22] observed from a satellite image, that is contains an assortment of features, surfaces and shadows and it can in this way be very complex to detect the ROI. The fascinating part of a satellite picture relies upon the application field for Earth perception. In this way image segmentation was developed for extracting distinctive features or surfaces inside the image

It is essential in image analysis to choose a satisfactory edge of gray level for removing objects from their background as proposed in [23]. Image thresholding is a straightforward, yet viable, method for dividing a picture into a foreground and background. This image examination method is a kind of image division that isolates objects by changing over grayscale pictures into binary pictures. Image thresholding is best in pictures with elevated amounts of contrast. Thresholding is the simplest and most commonly used method of segmentation [24]-[26].

In their research, [27] proposed advanced statistical and adaptive threshold techniques for video object detection and segmentation; the algorithms proposed depend on single-edge and three-outline differencing utilizing programmed and versatile factual thresholding strategies for motion object detection. Picture division also known as image segmentation is the way toward dividing a picture into parts or locales [8]. This division into parts is frequently in light of the attributes of the pixels in the picture. For instance, one approach to discover areas in a picture is to search for unexpected discontinuities in pixel esteems, which demonstrates edges. These edges can characterize areas. Another strategy is to partition the picture into districts in light of shading esteems, based on colour values. The goal of segmentation is to simplify and/or change the representation of an image into something that is more meaningful and less demanding to analyse. A decent segmentation is normally one in which [28]:

- Pixels in a similar class have comparable greyscale of multivariate values and form a connected region

- Neighbouring pixels which are in different classifications have different values.

Identifying and locating object in computerized image has turned out to be a standout amongst the most imperative applications for modern use to ease user and spare time. These techniques have been developed years back however improvement of it, is still required so as to accomplish the target more effectively and precisely.

\subsection{IMAGE HISTOGRAM DESCRIPTOR}

According to [29] the histogram of a digital image is an appropriation of its discrete intensity with $M$ total possible intensity levels in the range $[0, L-1]$. The distribution is a discrete function $h$ associating to every intensity level $r_{k}$ number of pixel with this intensity $n_{k}$. The function is as thus (Eq.(1)):

$$
h\left(r_{k}\right)=n_{k}
$$

where,

$r_{k}$ is the $k^{\text {th }}$ intensity level in the range[0,L-1].

$n_{k}$ is the number of pixels in the image whose intensity level is $r_{k}$ $L$ [ 255 for images of class uint8, 65535 for images class uint16 and 1.0 for images of class double].

The pixel values in an image can be represented by histograms. In this article histogram with 256 bins from gray image is we calculated.

\subsubsection{Transformation of Histogram:}

Normalization of a Histogram: Authors in [30] explained normalisation as a method comprising of changing the discrete distribution of intensities into a discrete distribution of probabilities. To do so, each value of the histogram is divided by the number of pixel (Eq.(2)).

$$
P\left(r_{k}\right)=h\left(r_{k}\right) / n=n_{k} / n, \text { for } k=1,2, . ., M
$$

where, $n$ is the total number of pixel.

Equalization of a Histogram: A technique to process images so as to modify the contrast of an image by adjusting the intensity distribution of the histogram [29]. The goal of this technique is to 
give a straight pattern to the cumulative probability function related to the image.

Let $f$ be a given image represented as a $m_{r}$ by mc matrix of integer pixel intensities ranging from 0 to $L-1$. $L$ is the number of possible intensity values, often 256 . Let $p$ denote the normalized histogram of $f$ with a bin for each possible intensity. So

$$
p_{n}=\frac{\text { number of pixels with intensity } n}{\text { total number of pixels }}
$$

where $n=0,1, \ldots, L-1$

The histogram equalized image $g$ is defined by (Eq.(3)):

$$
g_{i j}=\text { floor }\left((L-1) \sum_{n=0}^{f_{i j}} P_{n}\right)
$$

where floor() rounds down to the nearest integer.

\subsection{REGION DESCRIPTORS}

In [31] regions can either depict boundary based properties of an object or they can depict region based properties. It gauges a set of properties for each marked region $L$. $L$ can be a label matrix or a multidimensional array. At the point when $L$ is a label matrix, positive number components of $L$ correspond to various regions. The shape of an object alludes to its physical structure [32]. Region properties of an object gives you shape-based measurements of image regions. It's pretty useful, there are several supported measurements that are based on approximating regions by ellipses.

The shape is an important visual feature of an image [33]. Searching for objects using shape features has attracted much attention. There are many shape representation and description techniques in literature.

According to [32] shape is an important basic feature which is used to describe the image content. But because of the noise, occlusion, arbitrary distortion shape is often corrupted and object recognition problem became more complex. Shape representation is mainly based on the shape features which are either based on the shape boundary information or boundary plus interior content. Various types of shape features are designed for object recognition which are evaluated on the basis of how accurately those shape features allow one to retrieve the similar shapes from the database.

\subsection{SUPPORT VECTOR MACHINE}

As reported by [34] support vector machine (SVM) are classifiers, regressors and probability densities that is wellestablished in statistical learning hypothesis. SVM can be utilized for binary and multi-classification tasks. Authors used SVM classifier to classify vehicles into four categories namely; cars, jeeps, buses and trucks, separately, based on quantifiable image features.

\subsubsection{Binary Classification:}

SVM perform pattern recognition for two-class issues by deciding the separating hyperplane with greatest separation to the closest points of the training set. In this approach, ideal classification of a distinguishable two class issue is accomplished by boosting the width of the edge between the two classes [35]. The margin is the distance between the discrimination hyper- surface in $\mathrm{n}$ dimensional feature space and the nearest training patterns called support vectors. In the event that the data isn't linearly separable in the input space, a non-linear transformation $\Phi($.) can be applied, which maps the data points $x \in R$ into a high dimensional space $H$, which is known as a feature space. The data is then isolated as described previously. The first SVM was designed for linear separation of two classes; nonetheless, to tackle the problem of separating multiple classes, the multi-class SVM was created.

\subsubsection{Multi-Class Classification:}

SVM was intended to solve binary classification issues. In real world classification problems, however in any case, we can have multiple classes (more than two classes). In the endeavour to solve $q$ class problems with SVMs; training $q$ SVMs was involved.

Every one of which isolates a single class from all remaining classes, or preparing $q^{2}$ machines, every one of which isolates a pair of classes. Multi-class classification permits non-linearly separable classes by combining multiple 2-class classifiers. $\mathrm{N}$ class classification is accomplished by combining $\mathrm{N} 2$-class classifiers, each segregating between a particular class and the remainder of the training set [35]. Amid the classification stage, a pattern is assigned to the class with the largest positive distance between the classified patterns also, the individual separating hyper plane for the $\mathrm{N}$ binary classifiers. One of the two classes in such multi-class sets of binary classification issues will contain a considerably more modest number of patterns than the other class [35]. SVM classifier was picked in light of its popularity and ease of usage.

\section{PROPOSED METHOD}

This section introduces the novel technique for streetlight recognition, presenting the functionality of each module.

There have been multiple approaches to detect objects in the transport environment for example [36] proposed an algorithm approach to detect traffic sign boards from video sequences. However, none has been done to detect the streetlight which is a ubiquitous object on the road where autonomous vehicles operate.

Samples of original image, extracted streetlight and nonstreetlight image sets can be seen in Fig.1, Fig.2 and Fig.3.

A stationary camera was used to capture videos of dataset; the videos were then converted to frame to be able to acquire single images for processing and analysis. The Fig.1 shows samples of original image.

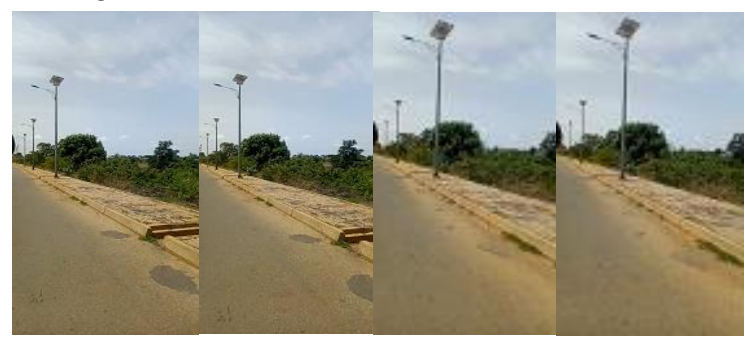

Fig.1. Samples of original images

The samples of original image are of size $1280 \times 720 \mathrm{px}$, containing both object to be detected and background. The images were captured from frames in a video before undergoing 
processing. ROI were manually extracted, to ensure noise is less for improved accuracy only the top area of the image containing the streetlight was cropped and are of size $197 \times 197$ pixels after cropping.

The Fig. 2 shows ROI images after cropping has been done to extract streetlight

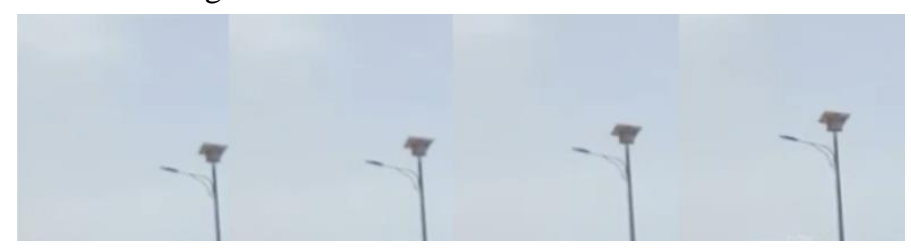

Fig.2. Samples of extracted streetlight images (Region of Interest)

Non streetlight Images were also extracted with size $197 \times 197$ pixels. The Non streetlight images contained objects not similar to the object to be detected. The Fig.3 shows non streetlight objects.

Fig.3. Samples of non-streetlight images

\subsection{STREETLIGHT OBJECT RECOGNITION}

The analysis of the performance of the proposed system for streetlight recognition was conducted on datasets gathered from camera of 13 Mega Pixels. The data used in the experimental analysis consisted of images, with the size $1280 \times 720$ px captured during daytime. The set of input-output example is computed as thus:

$$
\left(i_{1}, j_{1}\right),\left(i_{2}, j_{2}\right), \ldots,\left(i_{N}, j_{N}\right)
$$

where the input $i_{k}$ denotes the feature vector extracted from image patch $k$ and the output $j_{k}$ is a class label. Since we are categorising into streetlight and non-streetlight, the class label $y_{k}$ encodes streetlight and non-streetlight, respectively.

In [37] feature extraction is defined as a technique for capturing visual content of images for index and retrieval purposes. Primitive or low level image features can be either broad features, for example, extraction of colour, texture and shape or specific features. Feature extraction is performed on the ROI from the image input. MATLAB functions "imhist", "regionprops" and their combinations were used to represent streetlight and non-streetlight objects respectively.

\subsubsection{Region Descriptors/Features:}

We propose the use of 5 Region Features, which can be defined as follows as reported in [34].

1. Area: This is equivalent to the aggregate of pixels covered by the object. That is, Area of the object in a digital picture is the number of points in the object.

2. Solidity: Extent of the pixels in the convex hull that are additionally in the region.

3. Perimeter: Distance around the boundary of the region.
4. MinorAxisLength: Length (in pixels) of the minor axis of the ellipse that has indistinguishable normalized second moments from the region.

5. MajorAxisLength: Length (in pixels) of the major axis of the ellipse that has indistinguishable normalized second moments from the region. These five region properties were chosen because of their popularity and simplicity.

\subsubsection{Histogram Feature:}

The Histogram features were extracted as defined in section 4. Image histogram feature of 256 bins was computed thus:

$$
h\left(r_{k}\right)=n_{k}
$$

where, $r_{k}$ is the $k^{\text {th }}$ intensity level in the range $[0,255] . n_{k}$ is the number of pixels in the image whose intensity level is $r_{k}$.

\subsection{FEATURE COMBINATION}

In their work [38] stated that there are different procedures available to recognize object of a specific geometric shape from 2D picture. In any case, they are not very dependable techniques that distinguish features of objects of a picture and recognize the object having geometric shape like. These classical methods including histogram are very robust to some degree, but they are fallible and sometimes a combination of two features can improve efficacy, as the main goal for object detection is improved accuracy, as it portends great result.

In order to recognise, classify streetlight, we captured appearance and shape information using the proposed feature sets; in doing so, Region Features, and Image Histogram Features, defined in sections 4 and 5 were extracted from the segmented foreground object and were combined to form a feature attributes for the classification of the streetlight. The extracted Region (5), and Histogram (256) features were combined and used for the experiments.

\subsubsection{Flowchart of the Proposed System:}

The proposed system for streetlight detection follows the pseudocode:

Step 1: Video data of streetlights captured with a camera

Step 2: Video converted into frames of $1280 \times 720$ pixels

Step 3: Region Of Interest (ROI) of $197 \times 197$ pixels is cropped to avoid noise

Step 4: Segmentation using Graythresh is performed on images

Step 5: If appearance features are to be extracted whitespace is converted to colour foreground, then histogram features are extracted and classified using Linear SVM classifier, then streetlight object is detected

Step 6: The alternative is to extract 5 region features specifically: Area, Solidity, Perimeter, MinorAxisLength, MajorAxisLength and classified using Linear SVM classifier, then streetlight object is detected

Step 7: Extracted region and histogram features are combined and classified using Linear SVM classifier, then streetlight object is detected

Step 8: SVM with parameters: Kernel function = linear; Box constraint level $=1$; kernel scale mode = auto; standardised data. 
The Fig.4 illustrates the proposed streetlight recognition system in a block diagram.

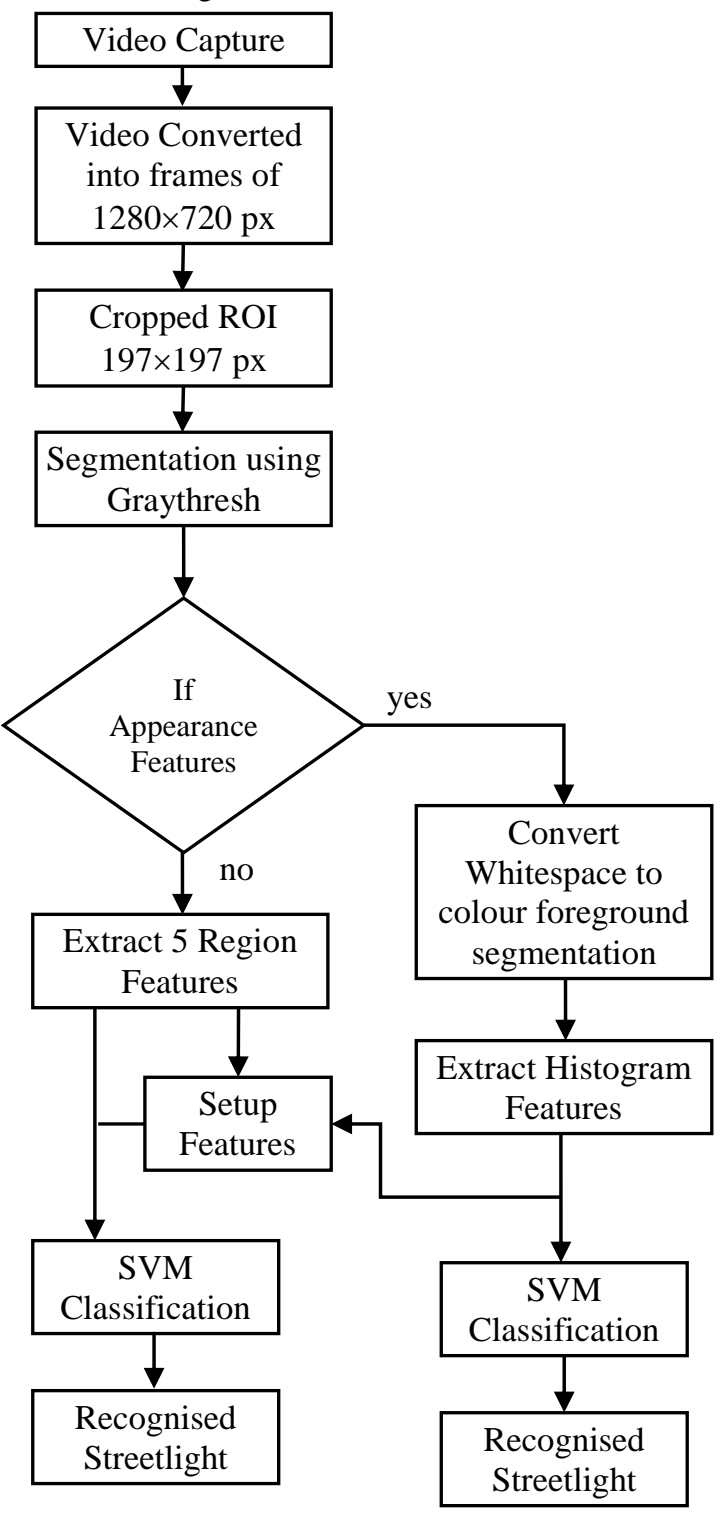

Fig.4. Flowchart of the proposed method

\section{EXPERIMENTAL ANALYSIS}

A number of experiments were conducted to evaluate the performance of the proposed algorithm on Streetlight object detection and recognition within daytime images. The experiments were conducted on images retrieved from dataset. The results obtain from these experiments are discussed in the following subsections.

The following are the parameters associated with the image dataset used to capture images. The dataset comprises images captured under daytime conditions.

A total of 667 streetlight image of dimension $197 \times 197$ were extracted. Similarly, a total of 1,680 background image sections with dimensions of $197 \times 197$ pixels were also extracted. For the purpose of classification, $60 \%$ of them were used for training and the remaining $40 \%$ for testing.
Table.1. Streetlight Camera Parameter

\begin{tabular}{|l|l|}
\hline Name & \multicolumn{1}{c|}{ Description } \\
\hline Sensor & Camera of 13 Mega Pixels, manually controlled \\
\hline \multirow{3}{*}{ Data } & $\bullet$ Total number of images $=2,347$ \\
& $\bullet$ Format of images $=$ RGB, \\
& $\bullet$ Image size $=1280 \times 720$ pixels \\
\hline
\end{tabular}

Samples of original image, extracted streetlight and nonstreetlight image sets can be seen in Fig.1, Fig.2 and Fig.3. Graythresh was applied on image, which was then complemented and then image is processed for feature extraction.

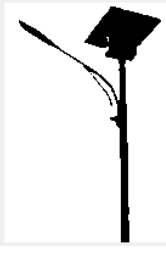

(a)

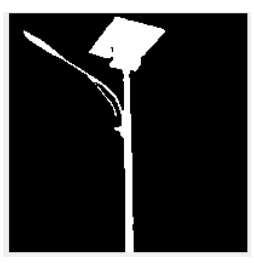

(b)

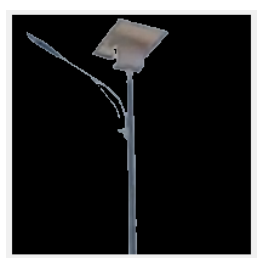

(c)
Fig.5. Samples showing (a) Graythresh image (b) Image complemented (c) Segmented streetlight after image processing

\section{RESULTS AND DISCUSSIONS}

A comprehensive performance evaluation was carried out of the proposed approach. The accuracy of the proposed technique is shown below:

Region descriptors performed the least with an accuracy rate of $84 \%$ due to inconsistencies of region being filled by the streetlight, histogram with $99.1 \%$ accuracy while combination of both had the highest accuracy highest accuracy rate of $100 \%$.

Table.2. Performance analysis on Streetlight Object detection

\begin{tabular}{|c|l|c|}
\hline Serial & \multicolumn{1}{|c|}{ Feature } & Accuracy (\%) \\
\hline 1 & Region Descriptors & $84 \%$ \\
\hline 2 & Histogram Descriptors & $99.1 \%$ \\
\hline 3 & Combination of 1 and 2 & $100 \%$ \\
\hline
\end{tabular}

The confusion matrix (Fig.6) and (Table.3) explains the level of accuracy graphically, and with values, it also shows instances where there has been wrong classification.

Class 1: Streetlight Object Class 2: Non-Streetlight Object

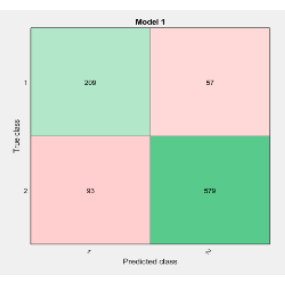

(a) Region Descriptor

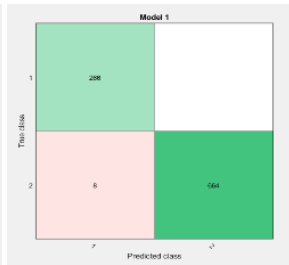

(b) Histogram Descriptor

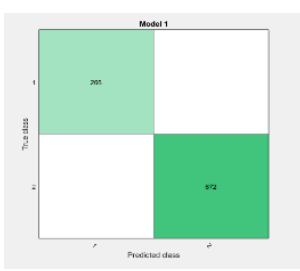

(c) Region and Histogram Combined
Fig.6. Confusion Matrix for the 3 different experiments performed 
Table.3. Confusion Matrix for the 3 different experiments performed

\begin{tabular}{|l|c|c|c|c|c|c|}
\hline & \multirow{2}{*}{ Feature } & Class 1 & Class 2 & \multicolumn{2}{c|}{$\begin{array}{c}\text { Correct } \\
\text { Prediction }\end{array}$} & \multicolumn{2}{c|}{$\begin{array}{c}\text { Wrong } \\
\text { Prediction }\end{array}$} \\
\cline { 4 - 7 } & & & $\mathbf{1}$ & $\mathbf{2}$ & $\mathbf{1}$ & $\mathbf{2}$ \\
\hline $\begin{array}{l}\text { Region } \\
\text { Descriptors }\end{array}$ & 266 & 672 & 209 & 579 & 57 & 93 \\
\hline $\begin{array}{l}\text { Histogram } \\
\text { Descriptors }\end{array}$ & 266 & 672 & 266 & 664 & - & 8 \\
\hline $\begin{array}{l}\text { Combination } \\
\text { of 1 and 2 }\end{array}$ & 266 & 672 & 266 & 664 & - & - \\
\hline
\end{tabular}

The Fig.7 shows a validated result of recognized streetlight from ten (10) randomly selected frames using the proposed histogram and region combined features that gave excellent result.
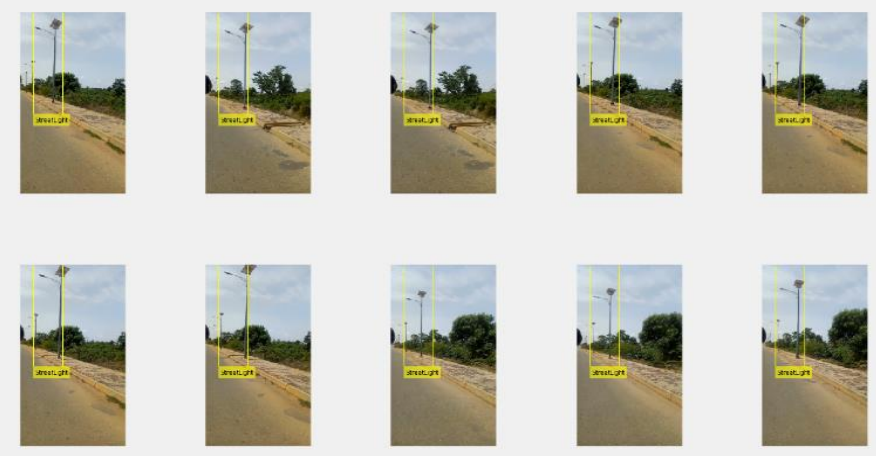

Fig.7. Validated result

For the purpose of detailed analysis of the performance of the proposed approach, the classification performance is evaluated using the receiver operating characteristic (ROC) curve (Fig.8) [34].

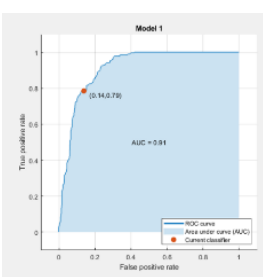

(a) Region

Descriptor

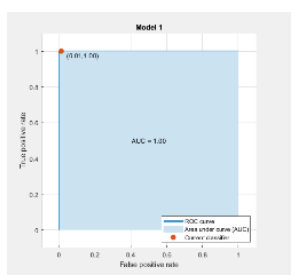

(b) Histogram Descriptor

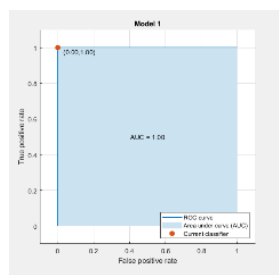

(c) Region and Histogram Combined
Fig.8. ROC curves for the 3 different experiments performed

From the ROC plot, all experiments performed were excellent with performance values of $91 \%, 100 \%$ and $100 \%$ respectively.

\section{CONCLUSION AND FUTURE WORK}

This paper proposed a feature-based technique for streetlight object detection and classification in daytime images. The features were extracted by applying histogram, and region on images. A linear SVM classifier was trained on the features obtained from the datasets (streetlights and non-streetlights). The proposed techniques were implemented and compared with the each other. Experimental results showed that Combination of
Region and Histogram outperformed with $100 \%$ accuracy, thereby exhibiting the highest detection and classification accuracy. Further experiments revealed that Histogram feature descriptors ranked second in performance with $99.1 \%$ accuracy. Future work could involve looking into identifying the streetlight in a live video footage.

\section{REFERENCES}

[1] S. Bagloee, M. Tavana, M. Asadi and T. Oliver, "Autonomous Vehicles: Challenges, Opportunities, and Future Implications for Transportation Policies", Journal of Modern Transportation, Vol. 24, No. 4, pp. 284-303, 2016.

[2] K. Waterloo, "Fairway Road Lanes Closed After Car Collides with Streetlight", Available at: https://www.cbc.ca/news/canada/kitchener-

waterloo/fairway-road-south-car-accident-1.4432176, Accessed on 2017.

[3] A. Rao, A. Angadi, B. Bhagyashree and R. Chaitra, "VisionBased Road Hump and Speed Breaker Detection", Available at:

http://www.kscst.iisc.ernet.in/spp/40_series/SPP40S/02_Ex hibition_Projects/183_40S_BE_1421.pdf

[4] H. Ward, "Night-Time Accidents, a Scoping Study", Available at: https://rospa.com/report/ nighttimeaccidents, Accessed on 2017.

[5] C. Atagi, "Busy Palm Springs Intersection Shutdown after Two Cars Collide, Knock Over Street Light", Available at: https://www.desertsun.com/story/news/traffic/2017/10/16/b usy-palm-springs-intersection-shutdown-after-two-carscollide-knock-over-street-light/768710001/, Accessed on 2017.

[6] C. Pocock, "Body, Face and Rectangle Detection: A Literature Review", Available at: https://people.cs.uct.ac.za/ jnorman/virpan/downloads/liter ature_review/literature_review-chris.pdf, Accessed on 2012.

[7] Y. Ramadevi, T. Sridevi, B. Poornima, and B. Kalyani, "Segmentation and Object Recognition using Edge Detection Techniques", International Journal of Computer Science and Information Technology, Vol. 2, No. 6, pp. 112, 2010.

[8] M.E. Irhebhude, "Object Detection, Recognition and ReIdentification in Video Footage", $\mathrm{PhD}$ Dissertation, Department of Information and Computing Science, Loughborough University, 2015.

[9] Mathworks, "Object Detection in Computer Vision", Available

at: https://www.mathworks.com/discovery/objectdetection.html, Accessed on 2018.

[10] N. Dalal and B. Triggs, "Histograms of Oriented Gradients for Human Detection", Proceedings of International Conference on Computer Vision and Pattern Recognition, pp. 886-893, 2005.

[11] S. Bindu, S. Prudhvi, G. Hemalatha, R. Sekhar and V. Nanchariah, "Object Detection from Complex Background Image using Circular Hough Transform", Journal of Engineering Research and Applications, Vol. 4, No. 4, pp. 23-28, 2014 
[12] M. Yadollahi and A. Prochazka, "Image Segmentation for Object Detection", Available at: http://dsp.vscht.cz/konference_matlab/MATLAB11/prispev ky/129_yadollahi.pdf

[13] S. Vijayalakshmi and C. Durairaj, "Use of Multiple Thresholding Techniques for Moving Object Detection and Tracking", International Journal of Computer Applications, Vol. 80, No. 1, pp. 12-18, 2013.

[14] A. Shubham and J. Singh, "Object Detection by Colour Threshold Method", International Journal of Advanced Research in Computer and Communication Engineering, Vol. 4, No. 10, pp. 1-9, 2015.

[15] K. Anusha and K. Sirisha, "Breaking the Speed Breakers using Image Processing", Available at: https://www.scribd.com/document/54957257/Breaking-theSpeed-Breakers-Using-Image-Processing-1-2, Accessed on 2011.

[16] R. Hussin, M. Juhari, N. Kang, R. Ismail and A. Kamarudin, "Digital Image Processing Techniques for Object Detection from Complex Background Image", Proceedings of International Conference on Robotics and Intelligent Sensors, pp. 167-174, 2012.

[17] A. Danti, J. Kulkarni, S. Jyothi and P.S. Hiremath, "A Technique for Bump Detection in Indian Road Images Using Color Segmentation and Knowledge Base Object Detection", International Journal of Scientific and Engineering Research, Vol. 4, No. 8, pp. 1-5, 2013.

[18] P. Kanubha and Y. Parmar, "A Research-Develop An Efficient Algorithm To Recognize, Separate and Count Indian Coin From Image Using Matlab", International Journal of Modern Trends in Engineering and Research, Vol. 3, No. 2, pp. 26-34, 2012.

[19] M.E. Irhebhude, M. Ali and E. Edirisinghe, "Pedestrian Detection and Vehicle Type Recognition", Proceedings of International Conference on Intelligent Computer Communication and Processing, pp. 1-5, 2015.

[20] R. Mallikka and M. Balamurugan, "Shape Based Feature Extraction in Detection of Image", Proceedings of International Conference on Computational Intelligence, pp. 1-6, 2018.

[21] R. Kate and S. Chitode, "Number Plate Recognition using Segmentation", International Journal of Engineering Research and Technology, Vol. 1, No. 9, pp. 41-47, 2012.

[22] M. Kiadtikornthaweeyot and A. Tatnall, "Region of Interest Detection based on Histogram Segmentation For Satellite Image", The International Archives of the Photogrammetry, Remote Sensing and Spatial Information Sciences, Vol. 11, No. 7, pp. 249-255, 2016
[23] N. Otsu, "A Threshold Selection Method from Gray-Level Histograms", IEEE Transactions on Systems, Man, and Cybernetics, Vol. 9, No. 1, pp. 62-66, 1979.

[24] N. Senthilkumaran and S. Vaithegi, "Image Segmentation by using Thresholding Techniques for Medical Images", Computer Science and Engineering: An International Journal, Vol. 6, No. 1, pp. 41-47, 2016.

[25] P. Kanungo, P. Kumar and S. Umesh, "Image Segmentation using Thresholding and Genetic Algorithm", Journal of Electrical and Computer Engineering, Vol. 2017, pp. 1-10, 2017.

[26] L. Christodoulou, K. Takis and O. Marques, "Advanced Statistical and Adaptive Threshold Techniques for Moving Object Detection and Segmentation", Proceedings of $3^{\text {rd }}$ International Conference on Digital Signal Processing, pp. 1-8, 2011.

[27] Segmentation, Available at: http://www.bioss.ac.uk/people/chris/ch4.pdf.

[28] A. Coste, "Histograms", Available at: http://www.sci.utah.edu/ acoste/uou/Image/project1/Arthur _COSTE_Project_1_report.html.

[29] S. Patel and P. Tandel, "A Survey on Feature Extraction Techniques for Shape based Object Recognition", International Journal of Computer Applications, Vol. 137, No. 6, pp. 16-20, 2016.

[30] D. Zhang and L. Guojun, "Review of Shape Representation and Description Techniques", Pattern Recognition, Vol. 37, No. 1, pp. 1-19, 2004.

[31] M.E. Irhebhude, A. Nawahda and E. Edirisinghe, "View Invariant Vehicle Type Recognition and Counting System using Multiple Features", International Journal of Computer Vision and Signal Processing, Vol. 6, No. 1, pp. 20-32, 2016.

[32] S. Milan, H. Vaclav and B. Roger, "Image Processing Analysis, and Machine Vision", $3{ }^{\text {rd }}$ Edition, CL Engineering, 2008.

[33] Y. Chincholkar and A. Kumar, "Traffic Sign Board Detection And Recognition For Autonomous Vehicles And Driver Assistance Systems", ICTACT Journal on Image and Video Processing, Vol. 9, No. 3, pp. 1954-1959, 2019.

[34] P. Mohanaiah, P. Sathyanarayana and L. Guru Kumar, "Image Texture Feature Extraction using GLCM", International Journal of Scientific and Research, Vol. 3, No. 5, pp. 1-8, 2013

[35] S. Patel, P. Trivedi, V. Gandhi and G. Prajapati, "Object Detection by Colour Threshold Method", International Journal of Advanced Research in Computer and Communication Engineering, Vol. 4, No. 10, pp. 1-9, 2013. 